\title{
Ecust004 Suppresses Breast Cancer Cell Growth, Invasion, and Migration via EMT Regulation
}

\author{
Ziyu Liu ${ }^{1,2}$ \\ Leilei Huang ${ }^{3,4}$ \\ Liwei Sun' \\ Hui $\mathrm{Nie}^{3,4}$ \\ Yuqi Liang ${ }^{1,2}$ \\ Jinwen Huang $\mathbb{1 D}^{3,4}$ \\ Fanhong $\mathrm{Wu}^{3,4}$ \\ Xin $\mathrm{Hu}^{1}$ \\ 'The Laboratory of Cancer Biology, \\ China-Japan Union Hospital of Jilin \\ University, Jilin University, Changchun, \\ jilin, People's Republic of China; \\ ${ }^{2}$ Department of Biochemistry and \\ Molecular Biology, School of Life Science, \\ Jilin University, Changchun, Jilin, People's \\ Republic of China; ${ }^{3}$ Shanghai Engineering \\ Research Center of Green \\ Fluoropharmaceutical Technology, \\ Shanghai, People's Republic of China; \\ ${ }^{4}$ Department of Pharmaceutical \\ Engineering, School of Chemical and \\ Environmental Engineering, Shanghai \\ Institute of Technology, Shanghai, People's \\ Republic of China
}

Correspondence: Xin Hu; Fanhong Wu Email huxin@jlu.edu.cn; wfh@sit.edu.cn
Purpose: Erianin is a small chemical compound extracted from Dendrobium chrysotoxum and has excellent antineoplastic effects against a variety of cancers. Combretastatin A-4 (CA4) is the most effective member of natural phenolic stilbene compounds isolated from the African willow tree Combretum caffrum. Ecust004 (Chemical Formula: $\mathrm{C}_{18} \mathrm{H}_{21} \mathrm{NO}_{7} \mathrm{~S}$ ) is a drug candidate optimized from structure-activity relationship studies of the sulfamate derivatives of Erianin and CA4, which has better bioavailability and pharmacokinetic profiles than Erianin and CA4.

Methods: To investigate the antitumor activity of Ecust004 in different types of breast cancer cells, MDA-MB-231 and MCF7 cells were treated with Ecust004. MTT and CCK8 were used to determine the effects of Ecust004 on cell proliferation. Wound-healing and Transwell assays were used to evaluate the migration and invasion level of cells treated with Ecust004. The expression of genes and proteins associated with epithelial-mesenchymal transition was detected by RT-PCR and Western blotting. In vivo studies further clarified the functional effects of Ecust004.

Results: Ecust004 treatment decreased the growth and proliferation of MDA-MB-231 and MCF7 cells at a lower dosage than Erianin. In addition, compared to Erianin and CA4, Ecust004 can better inhibit the invasion and migration of MDA-MB-231 and MCF7 cells. Accordingly, the expression of genes associated with epithelial-mesenchymal transition, such as E-cadherin and vinculin, was increased. Finally, compared with Erianin and CA4, Ecust004 exhibited a better anti-tumor activity in vivo.

Conclusion: Ecust004 inhibits the proliferation, invasion, and migration of breast cancer cells, and therefore represents a potential agent for development as an antitumor drug.

Keywords: Ecust004, Erianin, breast cancer, EMT

\section{Introduction}

Breast cancer is the most frequently diagnosed cancer in women. ${ }^{1}$ Surgery, chemotherapy, hormone therapy and radiation therapy are commonly used to treat breast cancer patients. ${ }^{2}$ However, these therapies are not effective for treating metastasis of breast cancer, which is the main cause of cancer-related mortality. Epithelial-mesenchymal transition (EMT) plays an essential role in the promotion of breast cancer metastasis. EMT is characterized with downregulation of the expression level of epithelial cell marker genes, including epithelial-cadherin (CDH1), and upregulation of the expression level of mesenchymal cell marker genes, including neuronal-cadherin (CDH2) and vimentin (VIM), which are regulated by multiple transcription factors such as zinc finger E-box-binding homeobox 1, 2 (ZEB1, ZEB2) and snail family transcriptional repressor 2 (SLUG). ${ }^{3-6}$ 
Furthermore, E-cadherin serves as an invasion suppressor that suppresses metastasis, and N-cadherin has been associated with the increased invasiveness of breast cancer cells. $^{7,8}$ The upregulation of EMT markers CDH2, Vim, SLUG, ZEB1 and ZEB2 is related to poor prognosis in breast cancer patients. ${ }^{9,10}$

Erianin is a small chemical compound extracted from the Chinese medicine Dendrobium chrysotoxum. ${ }^{11}$ Erianin demonstrates therapeutic potentials on various types of tumors. Erianin results in extensive tumor necrosis and inhibition of angiogenesis in hepatoma and melanoma cells. ${ }^{12}$ Furthermore, Erianin treatment inhibits the migration of T47D cells by regulating the steady-state expression of MPP and TIMP. ${ }^{13}$ In HL-60 cells, Erianin demonstrates potent inhibitory activity on cell proliferation, which is induced by apoptosis caused by the altered expression of Bax and Bcl-2 genes. ${ }^{14}$ In human osteosarcoma, Erianin induces autophagy, apoptosis, and cell cycle arrest through the ROS/JNK signaling pathway. ${ }^{15}$

Combretastins are natural phenolic stilbene compounds isolated from the bark of the African bush willow tree Combretum caffrum (Combretaceae). ${ }^{16}$ Combretastatin A-4 (CA4) is the most effective compound of these natural stilbenes. ${ }^{17}$ By inhibiting tubulin polymerization, CA4 increases vascular permeability and disrupts tumor blood flow. $^{18}$

Ecust004 is a drug candidate optimized from the structure-activity relationship studies of sulfamate derivatives of Erianin and CA4. It is a potent dual inhibitor on both tubulin and steroid sulfatase and shows strong antiproliferative activity on tumor cells at low nanomolar concentration. The sulfamate modification improves the bioavailability and pharmacokinetic profiles of the parent natural compounds. ${ }^{19-21}$ In this study, we evaluated the tumor inhibitory effects of Ecust004 on breast cancer cells and explored the molecular mechanisms underlying such effects.

\section{Materials and Methods}

\section{Chemicals}

Ecust004, Erianin, and CA4 were synthesized by Prof. Fanhong $\mathrm{Wu}$ (Shanghai Institute of Technology, Shanghai).

\section{Cell Culture}

MDA-MB-231 and MCF7 cells were kindly provided by the Cell Bank, Chinese Academy of Sciences. MDA-
MB-231 and MCF7 cells were maintained using Dulbecco's modified Eagle's medium (DMEM) with $10 \%$ fetal bovine serum and $100 \mathrm{IU} / \mathrm{mL}$ penicillinstreptomycin supplied by Solarbio. The MDA-MB-231 and MCF7 cell lines were cultured at $37{ }^{\circ} \mathrm{C}$ and $5 \%$ carbon dioxide.

\section{MTT Assays}

For the MTT assays, cells with different concentrations of Ecust004, Erianin, and CA4 were cultured in 96-well plates at 5000 cells per well for 48 hours. MTT (BestBio BB-4201-500T) solution was used to measure MDA-MB -231 and MCF7 cell proliferation. A volume of $10 \mu \mathrm{L}$ of $5 \mathrm{mg} / \mathrm{mL}$ MTT solution was added to 96-well plates, which was then cultured at $37^{\circ} \mathrm{C}$ in a $5 \% \mathrm{CO}_{2}$ atmosphere for 4 hours. The culture medium was removed after the treatment, and $150 \mu \mathrm{L}$ dissolving solution was added as provided by the kit (BestBio). A microplate reader (HBS1096; Detie) was used to measure the absorbance at 490 $\mathrm{nm}$. The conditions were repeated three times for each test.

\section{CCK8 Assays}

For the CCK8 assay, cells with different concentrations of Ecust004, Erianin, and CA4 were cultured in 96-well plates at 5000 cells per well for 72 hours. CCK8 (Bioss BA00208) solution were used to measure MDA-MB-231 and MCF7 cells proliferation. A volume of $10 \mu \mathrm{L}$ of CCK8 solution was added to 96-well plates, which was then cultured at $37^{\circ} \mathrm{C}$ at $5 \% \mathrm{CO}_{2}$ atmosphere for 4 hours. A microplate reader (HBS-1096; Detie) was used to measure the absorbance at $450 \mathrm{~nm}$. The conditions were repeated three times for each test.

\section{Immunofluorescence Staining}

MDA-MB-231 and MCF7 cells were seeded on slides and treated with Ecust004, Erianin, and CA4 for 24 hours. Cells were incubated in $4 \%$ formaldehyde at room for $15 \mathrm{~min}$ and $0.1 \%$ Triton $\mathrm{X}-100$ in PBS was added into the fixed cells for $20 \mathrm{~min}$. Next, $100 \mu \mathrm{L}$ of Phalloidin conjugate working solution (Abcam, ab176756; 1:1000) was added to each well of fixed cells. Cells were incubated at room temperature for 90 minutes. DAPI was used to stain DNA. The cytoskeleton (red) and DAPI (blue) were examined by fluorescence microscopy (Olympus IX51). 


\section{Wound-Healing Assays}

For the wound-healing assay, MDA-MB-231 and MCF7 cells were cultured in 6 -well plates at $6 \times 10^{5}$ cells per well upon reaching confluence. The cells were starved for 6 hours and $200 \mu \mathrm{L}$ sterile pipette tips were used to make scratches. Scratches were observed and photographed under fluorescence microscope (Olympus IX51) at $0 \mathrm{~h}$, $24 \mathrm{~h}$, and $48 \mathrm{~h}$ after scratching. Three images of each group were used to calculate the areas of scratch with Image J. The conditions were repeated three times for each test.

\section{Transwell Assay}

For the Transwell assay, MDA-MB-231 and MCF7 cells were starved for 6 hours. Matrigel (Corning 356234) was diluted in serum-free medium (1:3). The membranes of Transwell chambers (Corning 3422) were coated with $40 \mu \mathrm{L}$ diluted Matrigel at $37{ }^{\circ} \mathrm{C}$ for 1 hour and then $5 \times 10^{4}$ MDA-MB-231 and MCF7 cells were plated with serum-free medium. Inserts were placed in DMEM medium containing 20\% serum. After 48 hours, the cells on the upper layer of the chambers were removed. The cells that invaded on the lower layer of the chambers were fixed and stained with paraformaldehyde and Giemsa's stain (Solarbio G1010). Five Photos were taken of each group and the numbers of stained cells were counted.

\section{Cell Cycle Assay}

For the cell cycle assay, MCF7 cells were treated with Ecust004, Erianin, and CA4 for 48 hours. A total of $5 \times 10^{6}$ MCF7 cells were collected and stained with cell cycle detection kit (BestBio, BB-4104-3) at $4^{\circ} \mathrm{C}$ for 1 hour. The stained cells were detected by flow cytometry (BD FACSCalibur ${ }^{\mathrm{TM}}$ Cell Analyzer).

\section{Apoptosis Assay}

For the apoptosis assay, MCF7 cells were treated by Ecust004, Erianin, and CA4 for 48 hours. Next, $1 \times 10^{6}$ MCF7 cells were collected and stained with Annexin V-FITC apoptosis detection kit (BestBio, BB-4101-3). The stained cells were detected by flow cytometry (BD FACSCalibur ${ }^{\mathrm{TM}}$ Cell Analyzer).

\section{Quantitative RT-PCR}

For quantitative RT-PCR analysis, MDA-MB-231 and MCF7 cells were treated by Ecust004 for 48 hours. Total RNA was extracted with TaKaRa RNAiso Plus Reagent (TaKaRa, 9109), and cDNA was generated with TaKaRa PrimeScript ${ }^{\mathrm{TM}} \mathrm{RT}$ reagent kit and gDNA eraser (TaKaRa, RR047A). The expressions of CDH1, CDH2, ZEB1, ZEB2, VIM, CLDN3, and $\beta$-actin were analyzed by real-time PCR with the $2 \times$ EasyTaq $^{\circledR}$ PCR SuperMix (-dye) (AS111-01). Data were normalized against $\beta$-actin. mRNA expression levels were analyzed by paired $t$-test and results were represented as mean \pm SEM, with $\mathrm{p}<0.05$ as statistically significant difference. The sequences of real-time PCR primers are listed in Table 1.

\section{Western Blotting Assay}

For Western blotting assays, MDA-MB-231 and MCF7 cells were treated with Ecust004 for 48 hours. Cells were lysed in pre-cooled RIPA and the whole protein lysate was collected. The BCA Protein Assay Kit (Solarbio PC0020) was applied to determine the protein concentration. A total of $10 \mu \mathrm{g}$ protein of each group was separated on $10 \%$ Polyacrylamide gels under $140 \mathrm{~V}$ and transferred to PVDF blotting membrane. Next, 5\% skim milk was used as a blocking agent and to dilute primary antibodies (E-cadherin: CST 14472S, 1:1000; $\alpha$-catenin: Abcam ab51032, 1:1000; Slug: CST 9585S, 1:1000; vinculin

Table I Quantitative RT-PCR Primer Sequences (All Sequences from 5' to 3')

\begin{tabular}{|l|l|l|}
\hline RT-PCR Primers & Forward & Reverse \\
\hline ZEBI & GATGACCTGCCAACAGACCA & GCCCTTCCTTTCCTGTGTCA \\
ZEB2 & CCTCTGTAGATGGTCCAGTGAA & GTCACTGCGCTGAAGGTACT \\
CDHI & CAGTGAACAACGATGGCATT & CTGGGCAGTGTAGGATGTGA \\
CDH2 & CAATCCTCCAGAGTTTACTGCCATG & GATTGGTTTGACCACGGTGACTAAC \\
$V I M$ & ATTGAGATTGCCACCTACAG & ATCCAGATTAGTTTCCCTCAG \\
CLDN3 & GACCAACCTGCATGGACTGT & CGACGGGGTGGTCAAGTATT \\
$\beta$-actin & ACCAACTGGGACGACATGGA & GGTCTCAAACATGATCTGGGTCAT \\
\hline
\end{tabular}


[VCL]: Abcam ab18058, 1:1000; GAPDH: Bioworld AP0063, 1:5000), which was used to probe membranes overnight at $4{ }^{\circ} \mathrm{C}$. All the primary antibodies were detected with HRP-conjugated goat anti mouse or goat anti rabbit antibodies and developed using Chemiluminescent Analysis System (Tanon-5200 Tanon Science \& Technology).

\section{In vivo Assays}

Five-week old female BALB/c nude mice (Beijing Vital River Laboratory Animal Technology Co. Ltd, China) were injected with $1.0 \times 10^{7}$ MCF7 cells suspended in $100 \mu \mathrm{L}$ PBS per mouse into the flank. When the tumor size reached about $100 \mathrm{~mm}^{3}$, all mice were divided into four groups $(n=5)$ and treated with placebo, $50 \mathrm{mg} / \mathrm{kg}$ Ecust $004,50 \mathrm{mg} / \mathrm{kg}$ Erianin, or $50 \mathrm{mg} / \mathrm{kg}$ CA4 . All mice received an intraperitoneal injection every other day for a total of seven injections. Animals were weighed and the tumor size was measured at the indicated times and calculated as follows: length $(\mathrm{mm}) \times(\text { width }(\mathrm{mm}))^{2} / 2$. All animalrelated procedures were approved by the Animal Care and Use Committee of the Changchun Veishi Testing Technology Service (approval number: 20210528-01) and performed following the National Standard (GB/T 35892-2018) for Laboratory Animals Care and Use.

\section{Results}

\section{Ecust004 Inhibited the Growth of Breast Cancer Cells}

The chemical structure of Ecust004 is shown in Figure 1A. To study the effects of Ecust004, Erianin and CA4 on the proliferation, the viability of MDA-MB -231 and MCF7 cells was evaluated by CCK8 assays. Ecust004, Erianin, and CA4 inhibited the proliferation of MDA-MB-231 and MCF7 cells in a dosagedependent manner. Ecust004 caused a significant decrease in the viability of MCF7 and MDA-MB-231 cells at even $20 \mathrm{nM}$ (Figure 1B and E), while Erianin and CA4 started to decrease the viability of MCF7 and MDA-MB-231 cells at $40 \mathrm{nM}$ (Figure 1C, D, F and G), demonstrating that Ecust004 had a better inhibitory effect on cell proliferation than Erianin and CA4 at low doses. In addition, the viability of MDA-MB-231 and MCF7 cells treated with Ecust004, Erianin, and CA4 was detected by the MTT assay, which showed that Ecust004 was more effective on the inhibition of cell growth at a lower dosage (ie, $20 \mathrm{nM}$ ) than Erianin and CA4. (Figure S1). Higher concentrations of Ecust004 and Erianin were also used to treat MCF7 and MDA-MB-231 cells (Figure S2), and the result showed that higher concentrations of the drug did not lead to better inhibition.

\section{Ecust004 Suppressed the Migration of Breast Cancer Cells}

We first observed the morphology of breast cancer cells treated with Ecust004, Erianin and CA4. PhalloidiniFluor 555 staining was used to observe the cytoskeleton more clearly. While untreated and $20 \mathrm{nM}$ Erianin treated MCF7 cells demonstrated many mesenchymal features, MCF7 cells treated with Ecust004 showed some distinct epithelial cell morphology, such as a spherical-shaped appearance, indicating that Ecust004 altered the mobility of the treated cells (Figure 2A). Consistently, while untreated and $20 \mathrm{nM}$ Erianin treated MDA-MB-231 cells showed mesenchymal features, Ecust004 treatment suppressed these mesenchymal features (Figure 2B). Since Ecust004 changed the morphology of the breast cancer cells, we next investigated whether Ecust004 could affect MDA-MB-231 and MCF7 cell migration using wound healing assays. The migration of MCF7 cells (Figure 2C and D) and MDA-MB-231 cells (Figure 2E and F) treated with Ecust004, Erianin, and CA4 were suppressed compared with the negative control cells, and Ecust004 showed better inhibition of migration.

\section{Ecust004 Suppressed the Invasion of Breast Cancer Cells}

To investigate whether Ecust004 affected breast cancer cell invasion, MDA-MB-231 and MCF7 cells were treated with Ecust004, Erianin, and CA4 and tested using Transwell assays. Treatment with Ecust004, Erianin, and CA4 at 20 and $40 \mathrm{nM}$ reduced the invasion of MCF7 cells (Figure 3A and B). Similarly, treatment with Ecust004, Erianin, and CA4 at 20 and $40 \mathrm{nM}$ also suppressed the invasion of MDA-MB-231 cells (Figure 3C and D). Overall, in both types of breast cancer cells, Ecust004 had the strongest effect on inhibiting invasion compared with Erianin and CA4. 
A<smiles>COc1ccc(/C=C\c2ccc(OC)c(OS(N)(=O)=O)c2)cc1OC</smiles>
Ecust004

Chemical structure of Ecust004

$\mathrm{C}_{18} \mathrm{H}_{21} \mathrm{NO}_{7} \mathrm{~S} \quad \mathrm{Mr} 395.43$

B

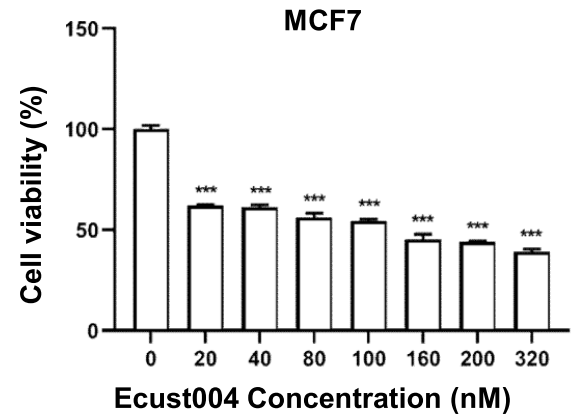

C

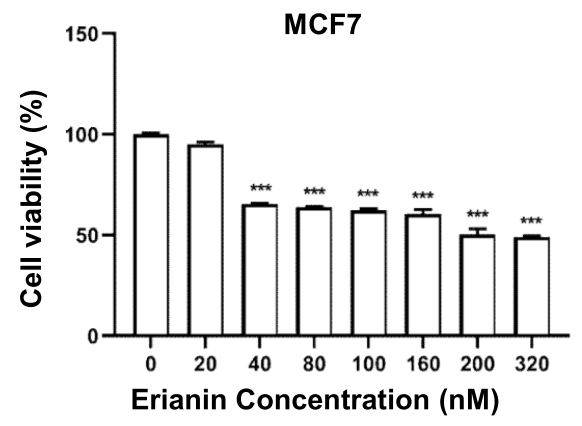

D

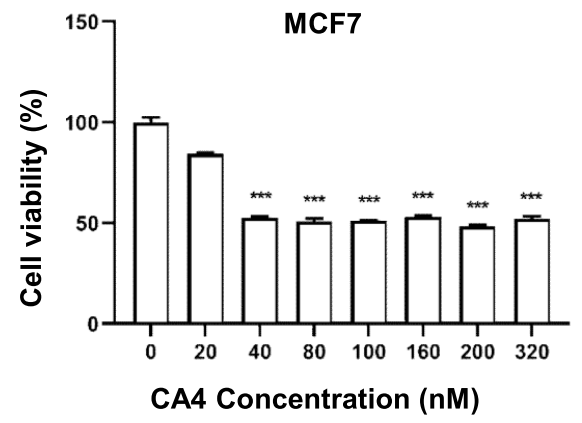

E

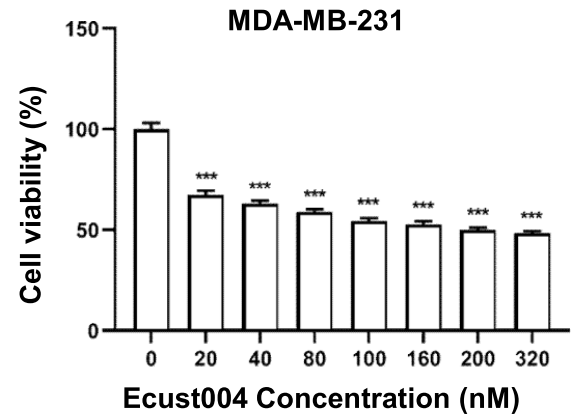

$\mathbf{F}$

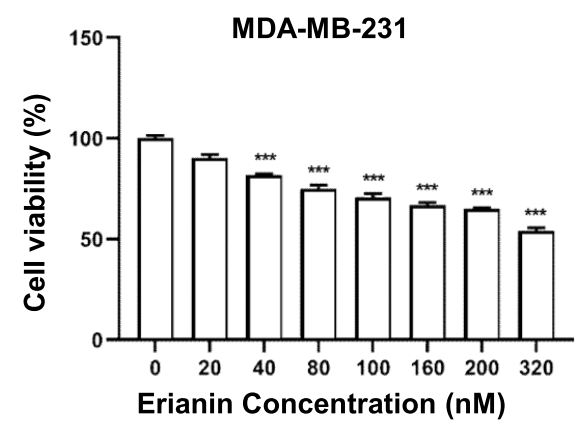

G

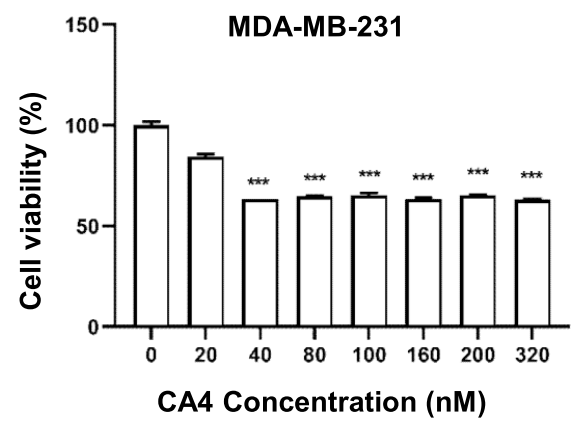

Figure I Ecust004 decreases the viability of MDA-MB-23I and MCF7 cells. (A) Chemical structure of Ecust004. Cell viability was determined using CCK8 assay after $72 \mathrm{~h}$ treatment with Ecust004 (B), Erianin (C) and CA4 (D) in MCF7 cells, and Ecust004 (E), Erianin (F) and CA4 (G) in MDA-MB-23I cells. The viability of untreated cells was set as $100 \%$. Data were compared using paired $t$-test and presented as mean \pm SEM, *** Indicates $p<0.00 \mathrm{I}(\mathrm{n}=3)$. 

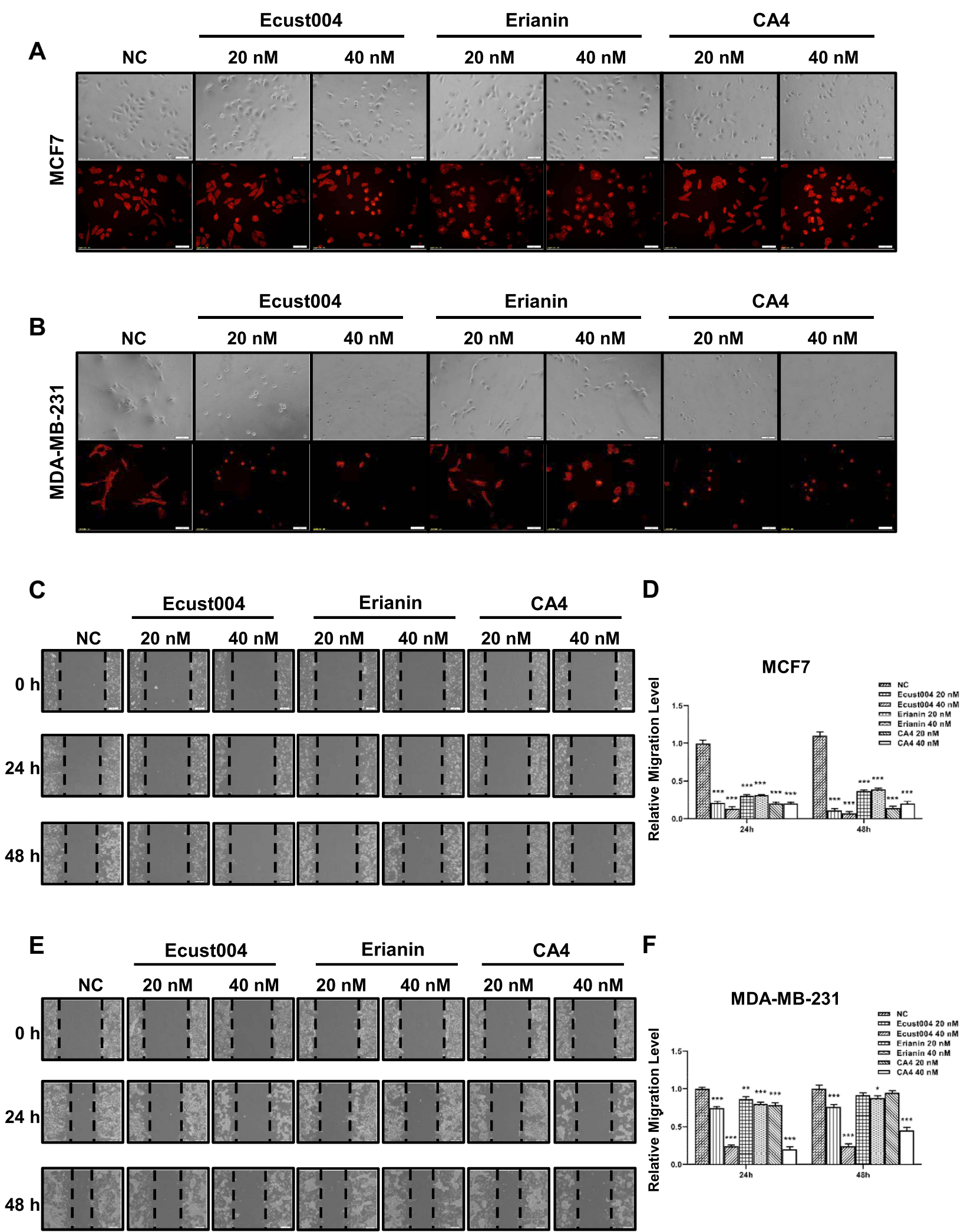

Figure 2 Ecust004 inhibits the migration of MDA-MB-23I and MCF7 cells. Representative images of the morphology of MCF7 (A) and MDA-MB-23I (B) cells treated with 20 and $40 \mathrm{nM}$ Ecust004, Erianin and CA4. Immunostaining showing the Phalloidin (red) labeled cytoskeleton. Scale bars represent $100 \mu \mathrm{m}$. Representative images of the MCF7 (C) and MDA-MB-23I (E) cells treated with 0, 20 and $40 \mathrm{nM}$ Ecust004, Erianin and CA4 in wound-healing assay. Scale bars represent I00 $\mu$ m. The relative migration level of MCF7 (D) and MDA-MB-23I (F) were quantified from (C and E). Data were compared using paired $t$-test and presented as mean \pm SEM, *Indicates $P<0.05$, **Indicates $\mathrm{p}<0.0 \mathrm{I}$, ****Indicates $\mathrm{p}<0.001(\mathrm{n}=9)$. 
A

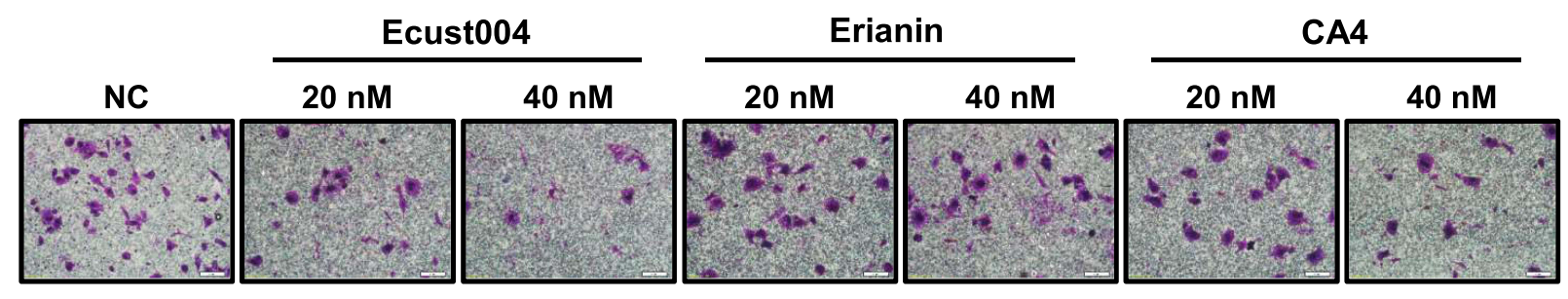

B

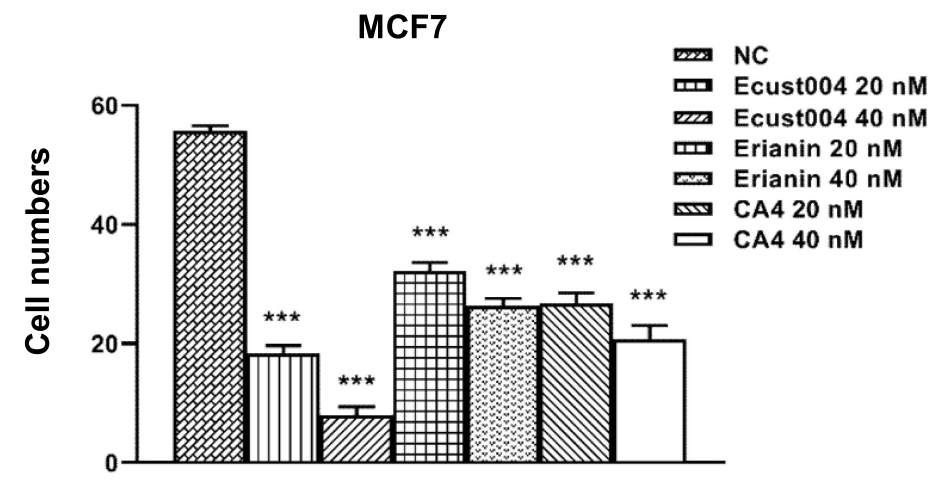

C

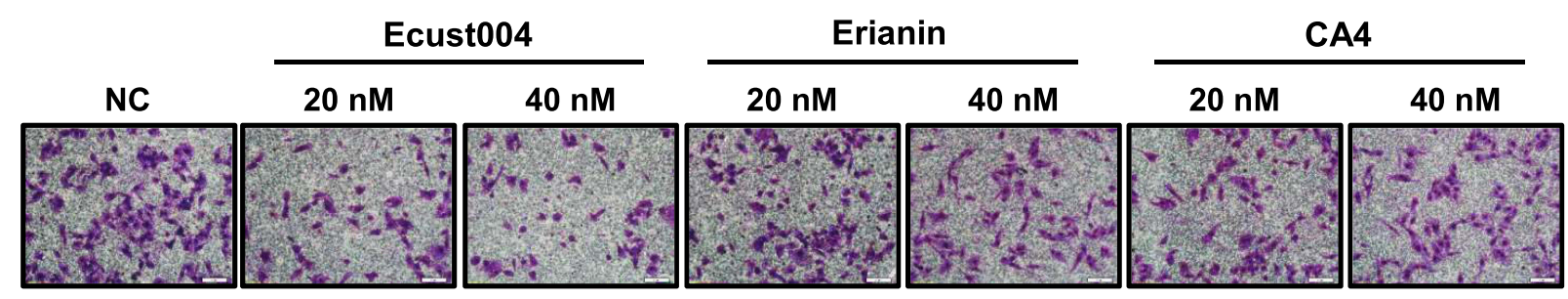

D

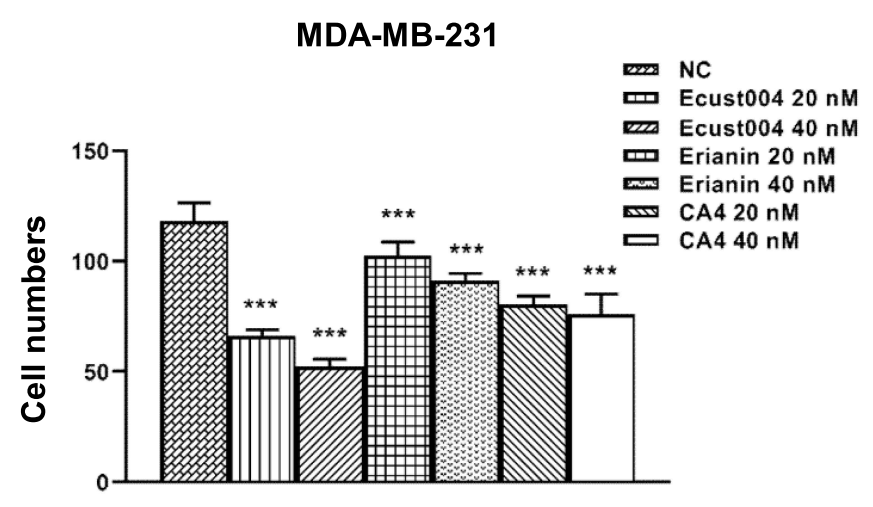

Figure 3 Ecust004 inhibits the invasion of MDA-MB-23I and MCF7cells. Representative images of the MCF7 (A) and MDA-MB-23I (C) cells treated with 0, 20, and 40 nM Ecust004, Erianin, and CA4 in Transwell assays. Scale bars represent $20 \mu \mathrm{m}$. The relative invasion level of MCF7 (B) and MDA-MB-23I (D) quantified in (A and C). Data were compared using paired $t$-test and presented as mean \pm SEM, ***indicates $p<0.001 \quad(n=5)$. 


\section{Ecust004 Induced Cell Cycle Arrest and Apoptosis of Breast Cancer Cells}

We also assayed the cell cycle and apoptosis of breast cancer cells treated with Ecust004, Erianin and CA4. The results showed that all three compounds increased the proportion of cells at the $\mathrm{G} 2 / \mathrm{M}$ phase (Figure S3A and B) and many cells treated with Ecust004, Erianin, and CA4 underwent apoptosis (Figure $\mathrm{S} 3 \mathrm{C}$ and $\underline{\mathrm{D}}$ ).

\section{Ecust004 Treatment Influenced the Expression of EMT Marker Genes}

To investigate the mechanisms underlying the inhibitory effects of Ecust004 on the invasion and migration of MDA-MB-231 and MCF7 cells, the expression of genes involved in EMT and cell adhesions were examined in MDA-MB-231 and MCF7 cells. RT-PCR results showed that Ecust004 treatment increased the transcription of CDH1 in MCF7 and decreased the transcription of CDH2, ZEB1, ZEB2, VIM, and CLDN3 in both MDAMB-231 and MCF7 cells. Since these genes were involved in the inhibition of EMT and promotion of cell adhesion, our results indicated that the inhibition on migration and invasion of Ecust004 was caused by the altered expression of genes that regulated EMT and cell adhesion (Figure 4A and B). Furthermore, the expression of these genes was examined by Western blotting. The expression of E-cadherin, VCL and $\alpha$ catenin was increased in MCF7 and MDA-MB-231 cells treated with Ecust004 (Figure 4C-F). The expression of SLUG, a transcription factor that promotes EMT process by inhibiting the expression of epithelial markers was decreased in both MCF7 and MDA-MB-231 cells treated with Ecust004 (Figure 4C-F). These results suggested that the inhibitory effect of Ecust004 on migration and invasion was caused by the decreased expression of SLUG.

\section{Ecust004 Inhibited Tumor Growth in vivo}

To investigate the effects of Ecust004 on tumors in vivo, we generated a xenograft tumor model using MCF7 cells and immune-compromised mice. MCF7 cells were injected subcutaneously into the mice to form tumors, and after 7 times of treatments with placebo, Ecust004, Erianin, or CA4, the tumor sizes and weight were analyzed from the treated mice. The tumor size and weight of the Ecust004 treated mice were significantly lower than the tumor size and weight of other three groups, which demonstrated that Ecust004 had much stronger inhibitory effects on the growth of tumors in vivo than the other chemicals (Figure 5A-C). In addition, the weights of the Ecust004 treated mice showed no significant changes with other mice, indicating that the treatment of Ecust004 did not affect the growth of mice (Figure 5D).

\section{Discussion}

Our previous study showed that Erianin inhibited the proliferation of tumor cells. ${ }^{13}$ However, due to its poor water solubility and low bioavailability, Erianin presents certain limitations as an anti-cancer treatment. The sulfamate modification is a well-established method to improve the bioavailability and pharmacokinetic profiles of chemical compounds, ${ }^{19-22}$ and Ecust004, the sulfamate modification of Erianin and CA4 presented improved bioavailability and pharmacokinetic profiles.

Our results indicated that the treatment of Ecust004 could inhibit the proliferation of tumor cells in vitro and in vivo. More importantly, Ecust004 could inhibit the cell viability, invasion, and migration of MDA-MB -231 and MCF7 cells at low dosages. Further research is needed to clarify the mechanisms involved in Ecust004 treatment inhibition of the growth and metastasis of breast cancer cells at lower dosage than Erianin.

The invasion and migration of MDA-MB-231 and MCF7 cells were significantly inhibited by the treatment of Ecust004. E-cadherin plays an important role as an invasion suppressor, suggesting that it should suppress metastasis. ${ }^{23} \alpha$-Catenin is an important protein in adhesions junctions and is responsible for maintaining intercellular adhesion and cellular polarity. ${ }^{24}$ Vinculin (VCL) is found in focal adhesion plaques, and is associated with the actin cytoskeleton in the process of ECM. ${ }^{25}$ SLUG has been reported to be a master transcription factor in regulating EMT. ${ }^{26}$ Interestingly, the expression of SLUG was decreased in MDA-MB-231 and MCF7 cells treated with Ecust004. Future investigation is needed to elucidate the mechanism involved in Ecust004-suppression of SLUG expression.

In conclusion, the sulfamate modification improved the inhibitory effects of Erianin on breast cancer cells; and thus, Ecust004 might be a potential candidate for the development as an antitumor agent. The effects of 

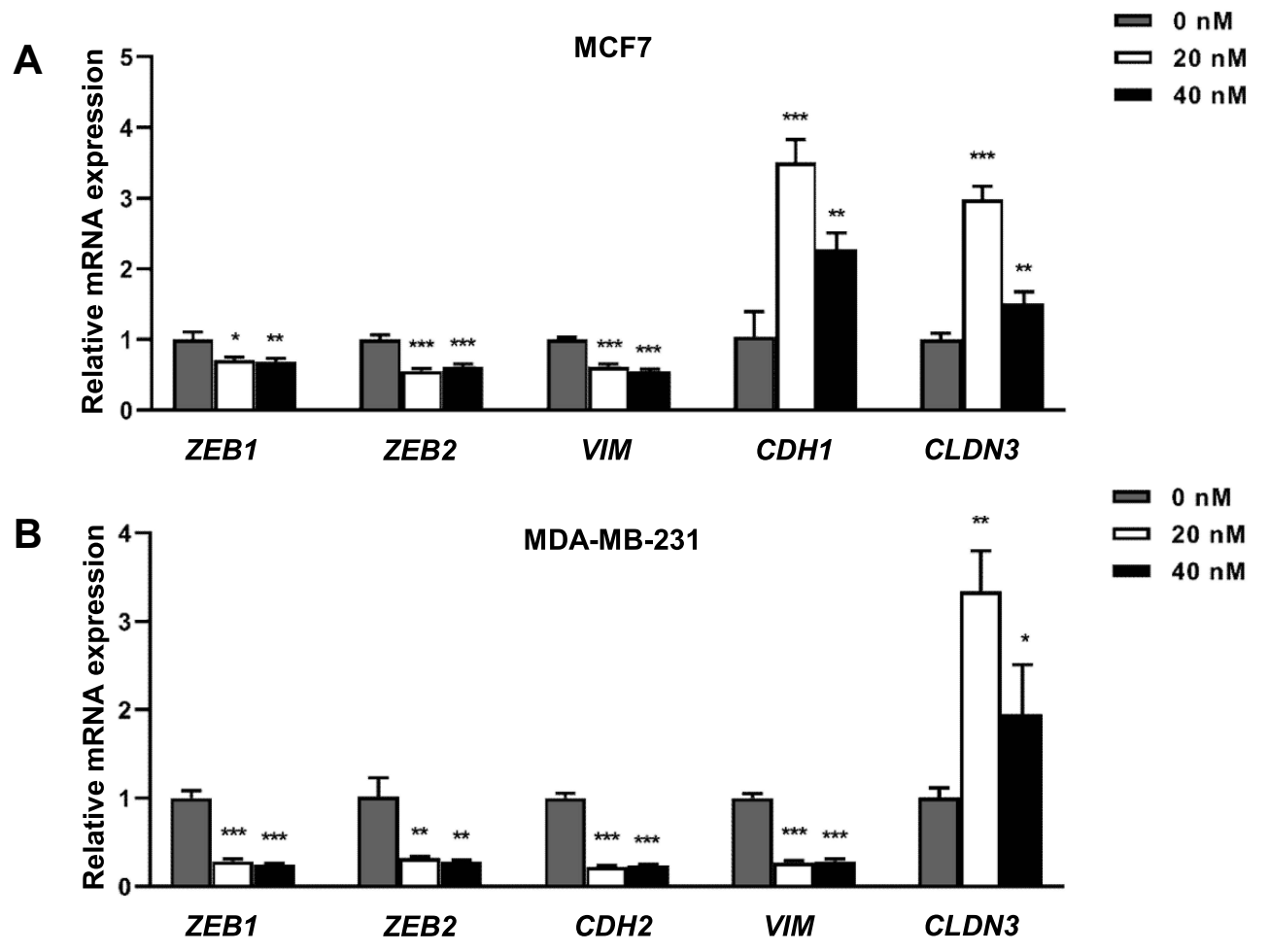

C

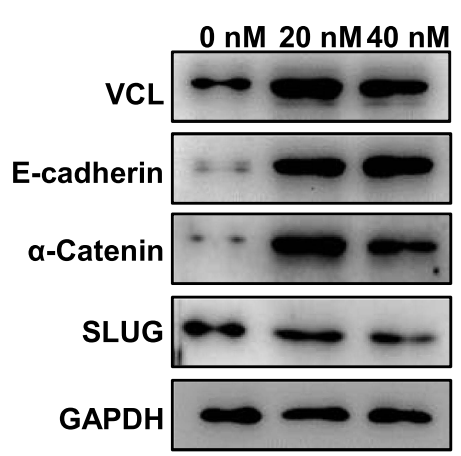

D

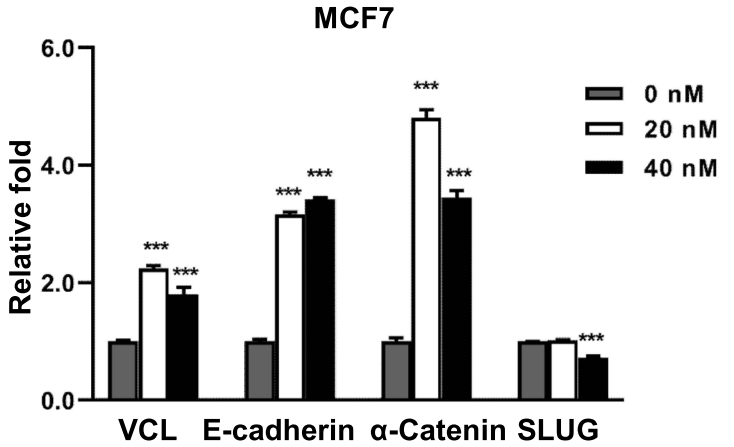

E

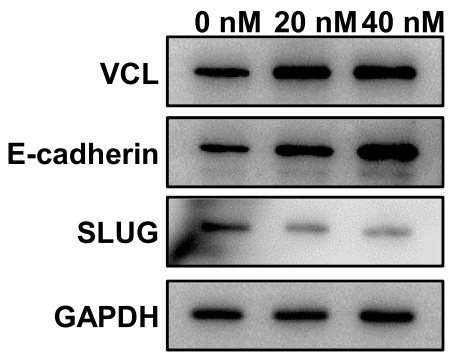

MDA-MB-231

F

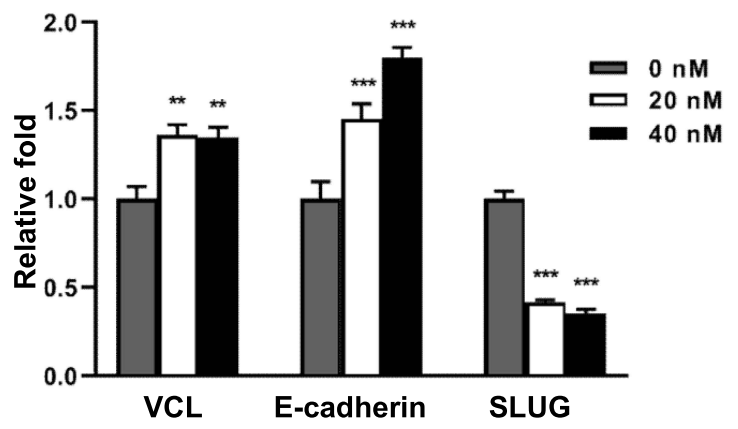

Figure 4 Ecust004 affects the expression of genes associated with EMT. (A) Real-time PCR analysis of the expression of ZEBI, ZEB2, VIM, CDHI and CLDN3 in MCF7 cells treated with 0, 20, and $40 \mathrm{nM}$ Ecust004. (B) Real-time PCR analysis of the expression of ZEBI, ZEB2, CDH2, VIM, and CLDN3 in MDA-MB-23I cells treated with 0, 20, and $40 \mathrm{nM}$ Ecust004. (C) Western blotting assays of the expression of VCL, E-cadherin, $\alpha$-catenin, SLUG, and GAPDH in MCF7 cells treated with 0 , 20 , and $40 \mathrm{nM}$ Ecust004. (D) Quantification of the expression of VCL, E-cadherin, $\alpha$-catenin, and SLUG from (C). (E) Western blotting assays of the expression of VCL, E-cadherin, SLUG, and GAPDH in MDA-MB-23I cells treated with 0, 20, and $40 \mathrm{nM}$ Ecust004. (F) Quantitation of the expression of E-cadherin, $\alpha$-catenin, and SLUG from (E). Data were compared using paired $t$-test and presented as mean \pm SEM, *Indicates $p<0.05$, **Indicates $p<0.01$, ***Indicates $p<0.00 I(n=3)$. 
A

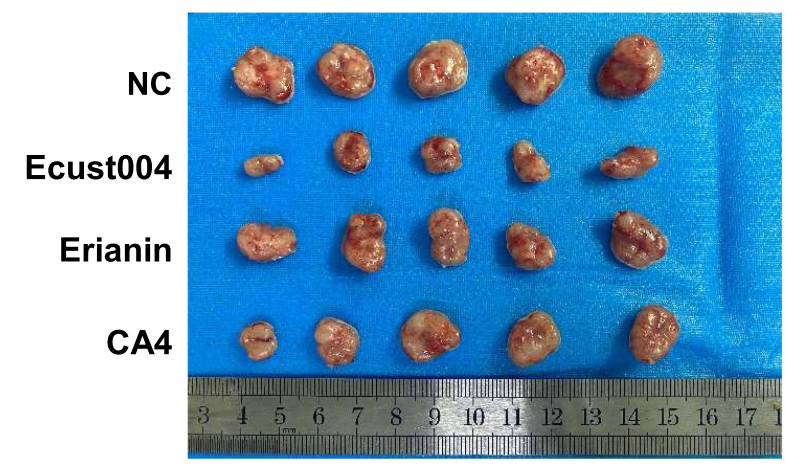

C

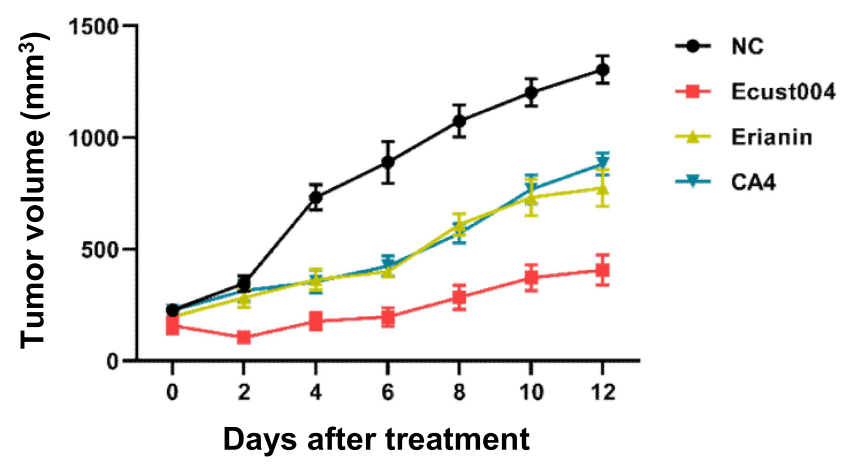

B

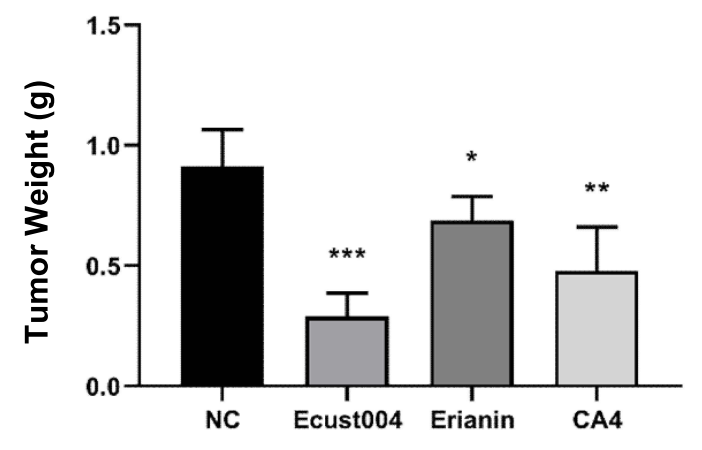

D

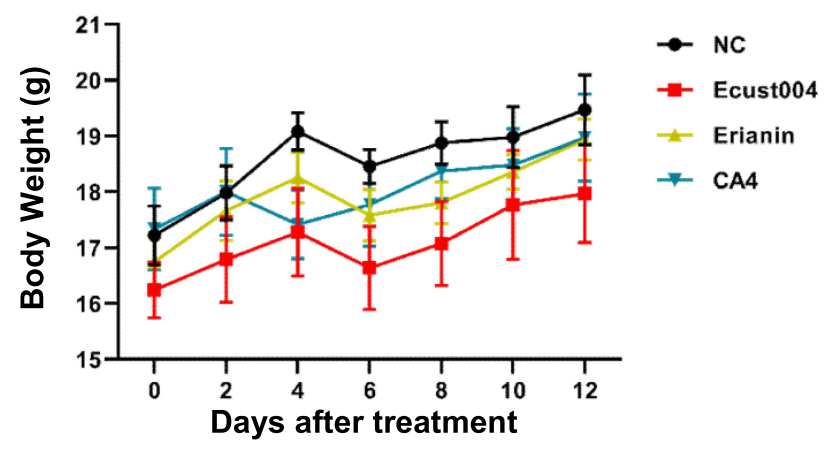

Figure 5 Ecust004 inhibits tumor growth in vivo. (A) Typical photos of tumors from the control and Ecust004, Erianin, CA4 treated groups. (B and C) tumor volume and weight of different groups. *Indicates $p<0.05$, **Indicates $p<0.01$, ***Indicates $p<0.001(n=5)$. (D) Mice body weights of controls and Ecust004, Erianin, CA4 treated groups was measure at indicated times.

Ecust004 on angiogenesis and autophagy need to be investigated in the future study.

\section{Data Sharing Statement}

All data generated and/or analyzed during this study are included in this published article.

\section{Acknowledgments}

This work was supported by grants from Jilin Province Science and Technology Development Project (20190701005GH, 20180101240JC), Jilin University Bethune Project (2020B13) and National Natural Science Foundation of China (NSFC, No. 21472126, 21302128, 21672151).

\section{Disclosure}

Dr Jinwen Huang reports a patent CN201710685120.3 licensed; Dr Fanhong $\mathrm{Wu}$ reports a patent
CN201710610734.9 issued. The authors declare no other conflicts of interest.

\section{References}

1. Siegel RL, Miller KD, Jemal A. Cancer statistics, 2020. CA Cancer J Clin. 2020;70(1):7-30. doi:10.3322/caac.21590

2. Waks AG, Winer EP. Breast cancer treatment: a review. JAMA. 2019;321(3):288-300. doi:10.1001/jama.2018.19323

3. Thiery JP, Sleeman JP. Complex networks orchestrate epithelial-mesenchymal transitions. Nat Rev Mol Cell Biol. 2006;7 (2):131-142. doi:10.1038/nrm1835

4. Schmalhofer O, Brabletz S, Brabletz T. E-cadherin, $\beta$-catenin, and ZEB1 in malignant progression of cancer. Cancer Metastasis Rev. 2009;28(1-2):151-166. doi:10.1007/s10555-008-9179-y

5. Berx G, Raspe E, Christofori G, Thiery JP, Sleeman JP. PreEMTing metastasis? Recapitulation of morphogenetic processes in cancer. Clin Exp Metastasis. 2007;24(8):587-597. doi:10.1007/ s10585-007-9114-6

6. Kong D, Li Y, Wang Z, Sarkar FH. Cancer stem cells and epithelial-tomesenchymal transition (EMT)-phenotypic cells: are they cousins or twins? Cancers. 2011;3(1):716-729. doi:10.3390/cancers30100716

7. Christofori G, Semb H. The role of the cell-adhesion molecule E-cadherin as a tumour-suppressor gene. Trends Biochem Sci. 1999;24(2):73-76. doi:10.1016/S0968-0004(98)01343-7 
8. Hazan RB, Phillips GR, Qiao RF, Norton L, Aaronson SA. Exogenous expression of $\mathrm{N}$-cadherin in breast cancer cells induces cell migration, invasion, and metastasis. J Cell Biol. 2000;148 (4):779-790. doi:10.1083/jcb.148.4.779

9. Eger A, Aigner K, Sonderegger S, et al. DeltaEF1 is a transcriptional repressor of E-cadherin and regulates epithelial plasticity in breast cancer cells. Oncogene. 2005;24(14):2375-2385. doi:10.1038/sj.onc.1208429

10. Comijn J, Berx G, Vermassen P, et al. The two-handed E box binding zinc finger protein SIP1 downregulates E-cadherin and induces invasion. Mol Cell. 2001;7(6):1267-1278. doi:10.1016/S1097-2765(01)00260-X

11. Liu H, Mu D, Lin T, Li Q. A simple method for the screening and measurement of phenols in Dendrobium chrysotoxum by a miniature mass detector using a matrix solid-phase dispersion method. J Anal Methods Chem. 2019;2019:6737632. doi:10.1155/2019/6737632

12. Gong YQ, Fan Y, Wu DZ, Yang H, Hu ZB, Wang ZT. In vivo and in vitro evaluation of erianin, a novel anti-angiogenic agent. Eur $J$ Cancer. 2004;40(10):1554-1565. doi:10.1016/j.ejca.2004.01.041

13. Sun J, Fu X, Wang Y, et al. Erianin inhibits the proliferation of T47D cells by inhibiting cell cycles, inducing apoptosis and suppressing migration. Am J Transl Res. 2016;8(7):3077-3086.

14. Li YM, Wang HY, Liu GQ. Erianin induces apoptosis in human leukemia HL-60 cells. Acta Pharmacol Sin. 2001;22(11):1018-1022.

15. Wang H, Zhang T, Sun W, et al. Erianin induces G2/M-phase arrest, apoptosis, and autophagy via the ROS/JNK signaling pathway in human osteosarcoma cells in vitro and in vivo. Cell Death Dis. 2016;7(6):e2247. doi:10.1038/cddis.2016.138

16. Pettit GR, Singh SB, Niven ML, Hamel E, Schmidt JM. Isolation, structure, and synthesis of combretastatins A-1 and B-1, potent new inhibitors of microtubule assembly, derived from Combretum caffrum. J Nat Prod. 1987;50(1):119-131. doi:10.1021/np50049a016

17. Pettit GR, Singh SB, Boyd MR, et al. Antineoplastic agents. 291. Isolation and synthesis of combretastatins A-4, A-5, and A-6(1a). J Med Chem. 1995;38(10):1666-1672. doi:10.1021/jm00010a011
18. Simoni D, Romagnoli R, Baruchello R, et al. Novel combretastatin analogues endowed with antitumor activity. J Med Chem. 2006;49 (11):3143-3152. doi:10.1021/jm0510732

19. Foster PA, Newman SP, Leese MP, et al. A new micronized formulation of 2-methoxyestradiol-bis-sulfamate (STX140) is therapeutically potent against breast cancer. Anticancer Res. 2008;28 (2A):577-581.

20. Raobaikady B, Reed MJ, Leese MP, Potter BV, Purohit A. Inhibition of MDA-MB-231 cell cycle progression and cell proliferation by C-2-substituted oestradiol mono- and bis-3-O-sulphamates. Int J Cancer. 2005;117(1):150-159. doi:10.1002/ijc.21066

21. Visagie MH, Birkholtz LM, Joubert AM. A 2-methoxyestradiol bis-sulphamoylated derivative induces apoptosis in breast cell lines. Cell Biosci. 2015;5:19. doi:10.1186/s13578-015-0010-5

22. Purohit A, Hejaz HA, Walden L, et al. The effect of 2-methoxyoestrone-3-O-sulphamate on the growth of breast cancer cells and induced mammary tumours. Int $J$ Cancer. 2000;85 (4):584-589. doi:10.1002/(SICI)1097-0215(20000215)85:4<584:: AID-IJC22>3.0.CO;2-Q

23. Padmanaban V, Krol I, Suhail Y, et al. E-cadherin is required for metastasis in multiple models of breast cancer. Nature. 2019;573 (7774):439-444. doi:10.1038/s41586-019-1526-3

24. Buckley CD, Tan J, Anderson KL, et al. Cell adhesion. The minimal cadherin-catenin complex binds to actin filaments under force. Science. 2014;346(6209):1254211. doi:10.1126/science.1254211

25. Demali KA. Vinculin-a dynamic regulator of cell adhesion. Trends Biochem Sci. 2004;29(11):565-567. doi:10.1016/j. tibs.2004.09.001

26. Chakrabarti R, Hwang J, Andres Blanco M, et al. Elf5 inhibits the epithelial-mesenchymal transition in mammary gland development and breast cancer metastasis by transcriptionally repressing Snail2. Nat Cell Biol. 2012;14(11):1212-1222. doi:10.1038/ncb2607
Drug Design, Development and Therapy

\section{Publish your work in this journal}

Drug Design, Development and Therapy is an international, peerreviewed open-access journal that spans the spectrum of drug design and development through to clinical applications. Clinical outcomes, patient safety, and programs for the development and effective, safe, and sustained use of medicines are a feature of the journal, which has also been accepted for indexing on PubMed Central. The manuscript management system is completely online and includes a very quick and fair peer-review system, which is all easy to use. Visit http://www. dovepress.com/testimonials.php to read real quotes from published authors. 